\title{
Warps in Galactic Disks
}

\author{
Gyula I.G. Józsa ${ }^{1}$, Franz Kenn $^{1}$, Ulrich Klein ${ }^{1}$ \\ and Thomas A. Oosterloo ${ }^{2,3}$ \\ ${ }^{1}$ Argelander Institute for Astronomy (AIfA), Univ. Bonn \\ Auf dem Hügel 71, D-53121 Bonn, Germany \\ ${ }^{2}$ Netherlands Foundation for Research in Astronomy \\ Postbus 2, 7990 AA Dwingeloo, The Netherlands \\ ${ }^{3}$ Kapteyn Institute, Univ. of Groningen \\ P.O. Box 800, 9700 AV Groningen, The Netherlands
}

\begin{abstract}
We have embarked on a detailed study of the kinematical and morphological structure of HI disks of warped galaxies, which are parametrised by means of kinematic modelling of spectroscopic observations. We present current results and compare them to predictions of theories for warp formation and -maintenance.
\end{abstract}

Keywords. galaxies: ISM, structure, kinematics and dynamics, methods: data analysis

\section{Introduction}

Probably every galaxy with a gaseous disk extending beyond the optical one shows a warp. Warps in denser environments tend to be more asymmetric and to have a larger amplitude (García-Ruiz 2002). This hints of excitation mechanisms, where warps are the results of tidal interactions. But also isolated galaxies show symmetric warps with high amplitudes (e.g. NGC 5055, Battaglia et al. 2006) and the number density of detected warps does not seem to depend significantly on the visible environment. Especially for these, a - possibly different - mechanism must exist that prevents a winding-up and destruction of the warp through differential precession. Such warps are long-lived and/or they are frequently generated. The latter would then require the interaction with invisible agents. Recent theoretical studies link the excitation of warps to the late infall of extragalactic material. Warps might be excited frequently or continuously as a reaction on the late infall of dark material (Ostriker \& Binney 1988, Shen \& Sellwood 2006) or the warped material itself consists of formerly accreted gas (van der Kruit 2007).

Here, we present results of our ongoing study of the kinematical properties of symmetric warps. A detailed description of the data and the analysis can be found in Józsa (2007).

\section{Observations and analysis technique}

In the scope of the study presented here we selected the galaxies NGC 5204, UGC 3580, and NGC 2541 as suitable candidates with distinctly symmetric H I disks. The WSRT observations in the $\mathrm{H}$ I emission line resulted in high quality data cubes.

We developed a software tool called TiRiFiC (Tilted-Ring-Fitting-Code) in order to parametrise $\mathrm{H}$ I disks of galaxies in terms of a tilted-ring model and applied the software to the obtained data cubes. TiRiFiC produces synthetic observations that are compared directly to input data cubes. The parametrisation is automatically optimised via $\chi^{2}-$ minimisation. With this technique we are not hampered by systematics caused by the 
crowding of orbits along the line-of-sight as occur in analyses of velocity fields (see Corbelli \& Schneider 1997 and Józsa et al. 2007).

\section{Results}

We confirm Briggs' (1990) rules:

i) Within $r_{25}$ the (H I-)disk is planar

ii) The warp commences where the stellar disk fades. The line-of-nodes (LON) is defined as the projection of the tip of the spin normal vector of the disk onto the plane of the inner disk. This LON remains straight out to a transition radius $r_{\mathrm{tr}}$. Beyond $r_{\mathrm{tr}}$ the LON forms a spiral that is loosely wound in the direction of the galactic rotation Furthermore we observe:

iii) At large radii, the orientation of the disk becomes constant again. The galaxy shows a two-disk structure, consisting of an inner disk aligned with the stellar body, and a more or less flat outer disk, the warp being the transition from one orientation to another

iv) Under the assumption of circular orbits, a change of the rotation velocity occurs at the transition radius (with varying significance)

v) The HI surface-density drops markedly at the transition radius, beyond which it exhibits a shallow decrease

\section{Discussion}

The findings presented here have been reported in the literature for a number of other galaxies (see e.g. Corbelli \& Schneider 1997, van der Kruit 2007, and Józsa 2007). Therefore, they may be typical for evolved, symmetric warps. The structure of the analysed H I disks, viz their outer flat regime, is reminiscent of the galaxy structure found in cosmological simulations by Bailin et al. (2006): after a last merger a galaxy quickly settles into a long-lived intrinsic misalignment with the flattened Dark Matter halo; the outer portion of the halo is inclined with respect to the inner disk-Dark Matter halo system, which itself is well aligned. A tenuous H I component would trace the flat Dark Matter halo at large radii. The scenario proposed by Shen \& Sellwood (2006), simulating cosmic infall with an external ring of growing (and decreasing) mass, could - within the errors also be compatible with our findings. They predict as a major feature a change of the direction of the spirality of the LON (a twist) at large radii. While being incompatible with a purely flat outer disk, an elongated twist region could mimic a flat disk.

\section{References}

Bailin, J., Kawata, D., Gibson, B. K., et al. 2005, ApJ, 627, L17

Battaglia, G., Fraternali, F., Oosterloo, T. \& Sancisi, R. 2006, A\&A, 447, 49

Briggs, F. H. 1990, ApJ, 352, 15

Corbelli, E. \& Schneider, S. E. 1997, ApJ, 479, 244

García-Ruiz, I., Sancisi, R. \& Kuijken, K. 2002, A\&A, 394, 769

Józsa, G. I. G., Kenn, F., Klein, U. \& Oosterloo, T. A. 2007, A\&A, 468, 731

Józsa, G. I. G. 2007, A\&A, 468, 903

van der Kruit, P. C. 2007, A\&A, 466, 883

Ostriker, E. C. \& Binney, J. J. 1989, MNRAS, 237, 785

Shen, J. \& Sellwood, J. A. 2006, MNRAS, 370, 2 